\title{
BOUNDED AND CONTINUOUS RANDOM FOURIER SERIES ON NONCOMMUTATIVE GROUPS
}

\author{
ALESSANDRO FIGÀ-TALAMANCA
}

1. Introduction. The following two results were proved by $P$. Billard:

I. Let $\xi_{n}(n= \pm 1, \pm 2, \cdots)$ be independent random variables equidistributed on the interval $(0,2 \pi]$. Consider the random Fourier series

$$
\sum_{n=-\infty}^{+\infty} a_{n} \exp \left[i n\left(x+\xi_{n}\right)\right],
$$

where $a_{n}$ are fixed complex numbers. Then if (1) represents with probability one a function in $L^{\infty}(0,2 \pi)$, it represents with probability one a continuous periodic function [1, Proposition A].

II. Let $\epsilon_{n}$ be independent random variables taking the values +1 and -1 with equal probability. Consider the random Fourier series

$$
\sum_{n=-\infty}^{+\infty} \epsilon_{n} a_{n} \exp (i n x)
$$

where $a_{n}$ are fixed complex numbers. Then if (2) represents with probability one a function in $L^{\infty}(0,2 \pi)$, it represents with probability one a continuous periodic function [2, Théorème 1].

The purpose of this note is to show that while I holds, when appropriately interpreted, for Fourier series on arbitrary compact groups, II does not hold in general if the group is noncommutative.

The fact that a generalization of I holds for arbitrary groups follows easily from Billard's original proof and some results of Kahane on random series in Banach spaces [4, Théorème 2] and [5, Chapter II, Theorem 1]. With the help of another result of Kahane [4, Theorème $3]$ and [5, Chapter II, Theorem 5] one can easily show that II holds for Fourier series defined on an arbitrary commutative compact group. Our example in $\S 3$ shows that II can fail at least for some noncommutative groups.

One should notice that II is a stronger result than $I$, in fact an application of Fubini's theorem yields that if II is true, the conclusion also holds for random Fourier series of the type

Presented to the Society, January 24, 1969 under the title Randomly continuous Fourier series on compact groups; received by the editors January 3, 1969. 


$$
\sum_{n=-\infty}^{+\infty} X_{n} \exp (i n x)
$$

where $X_{n}$ are independent symmetric complex valued random variables (symmetric means that $X_{n}$ and $-X_{n}$ are similar) [2, Théorème $6]$.

2. Billard's theorem for compact groups. Let $G$ be a compact group. Let $\left\{D_{n}\right\}_{n=1}^{\infty}$ be a sequence of nonequivalent irreducible unitary representations of $G$ and let $d_{n}$ be the degree of $D_{n}$. Let $\xi_{n}$ be a sequence of independent random variables with values on $G$. We assume that $\xi_{n}$ are equidistributed, i.e. that the measure induced by each of them on $G$ is the Haar measure. Let $\epsilon_{n}$ be independent random variables, also independent of the $\xi_{n}$, taking with equal probability, the values +1 and -1 . We shall call the $\epsilon_{n}$ the Bernoulli random variables. Let $A_{n}$ be fixed $d_{n} \times d_{n}$ matrices. We consider the random Fourier series

$$
\sum_{n=1}^{\infty} \epsilon_{n} d_{n} \operatorname{tr}\left(A_{n} D_{n}\left(\xi_{n} x\right)\right)
$$

We want to prove the following

THEOREM. If (4) represents with probability one a function in $L^{\infty}(G)$, then it represents with probability one a continuous function.

For the proof we use the following result of Kahane [4, Théorème 2] and [5, Chapter II, Theorem 1].

LeMмA 1. Let $X_{n}$ be independent random variables with values in a Banach space. Suppose that for each $n,-X_{n}$ and $X_{n}$ are similar (i.e. they have the same probability distribution). Let $S=\left\{a_{n m}\right\}$ be an infinite matrix satisfying $\lim _{n} a_{n m}=1$. Then if the event defined by the relations

$$
\begin{aligned}
& \limsup _{p, p^{\prime} \rightarrow \infty}\left\|\sum_{m=p}^{p^{\prime}} a_{n m} X_{m}\right\|=0 \quad(n=1,2, \cdots) \\
& \underset{n \rightarrow \infty}{\limsup } \limsup _{p \rightarrow \infty}\left\|\sum_{m=1}^{p} a_{n m} X_{m}\right\|<\infty,
\end{aligned}
$$

has probability one, the event defined by the relation

$$
\underset{p \rightarrow \infty}{\lim \sup }\left\|\sum_{m=1}^{p} X_{m}\right\|<\infty
$$

also has probability one. 
COROLlaRY. If (4) represents with probability one a function in $L^{\infty}$, then the event

$$
\underset{p \rightarrow \infty}{\limsup }\left\|\sum_{n=1}^{p} \epsilon_{n} d_{n} \operatorname{tr}\left(A_{n} D_{n}\left(\xi_{n} x\right)\right)\right\|_{\infty}<\infty
$$

has probability one.

Proof. Let $k_{\alpha}$ be a central approximate identity of $L^{1}(G)$ consisting of trigonometric polynomials. That is (i) $k_{\alpha}$ has a finite Fourier series development, (ii) $\left\|k_{\alpha}\right\|_{1} \leqq 1$ and $\lim _{\alpha}\left\|k_{\alpha} * f-f\right\|_{1}=0$, for every $f \in L^{1}$. Since the closed ideal of $L^{1}$ generated by the characters of the representations $\left\{D_{n}\right\}_{n=1}^{\infty}$ is separable, we can choose a sequence $\left\{k_{n}\right\}$ $\subseteq\left\{k_{\alpha}\right\}$ such that $\lim _{n}\left\|k_{n} * f-f\right\|_{1}=0$ for every $f$ belonging to this ideal. Let $e_{n}$ be the character of $D_{n}$ and let $k_{n} * e_{m}=a_{n m} e_{m}$. Then $\lim _{n} a_{n m}=1$. If $f w(\omega \in \Omega$, the probability space on which the random variables are defined) is the $L^{\infty}$ function represented by (4), then

$$
k_{n} * f_{\omega}=\sum_{m=1}^{\infty} \epsilon_{m} a_{n m} d_{m} \operatorname{tr}\left(A_{m} D_{m}\left(\xi_{m} x\right)\right),
$$

where all but finitely many coefficients $a_{n m}$ are zero. Thus $k_{n} * f_{\omega}$ $\in C(G)$ and $\left\|k_{n} * f_{\omega}\right\|_{\infty} \leqq\left\|k_{n}\right\|_{1}\left\|f_{\omega}\right\|_{\infty}$; therefore the series (5) satisfies the hypothesis of the lemma and the conclusion follows.

Proof of THE TheOREM. What follows is nothing but a translation in the framework of arbitrary groups of Billard's proof of Proposition A of [1]; the Corollary just proved will be used instead of Lemma 3 of [1]. Suppose that with positive probability (4) does not represent a continuous function, then the argument given in [1, Lemma 5] or [5, Theorem 1] shows that there exist $\epsilon>0$ and numbers $m_{k}, M_{k}$ such that $m_{k}<M_{k}<m_{k+1}$ and

$$
\operatorname{Pr}\left\{\left\|\sum_{n=m_{k}}^{M_{k}} \epsilon_{n} d_{n} \operatorname{tr}\left(A_{n} D_{n}\left(\xi_{n} x\right)\right)\right\|_{\infty}>\epsilon\right\}>\epsilon .
$$

Let $U_{k}=\sum_{n=m_{k}}^{M_{k}} \epsilon_{n} d_{n} \operatorname{tr}\left(A_{n} D_{n}\left(\xi_{n} x\right)\right)$, then by the reasoning made in $[6$, p. 220],

$$
\sum_{k=1}^{\infty} U_{k} \in L^{\infty} \quad \text { with probability one. }
$$

But then, applying the Corollary to the series in (6) we obtain

$$
\lim \sup _{\nu}\left\|\sum_{k=1}^{p} U_{k}\right\|_{\infty}<\infty \quad \text { with probability one. }
$$


Let $\Omega$ be the probability space on which $\epsilon_{n}$ and $\xi_{n}$ are defined. We introduce probability spaces $\Omega^{\prime}$ and $\Omega^{\prime \prime}$ and random variables $\epsilon_{k}^{\prime}$ and $\xi_{k}^{\prime \prime}$ defined in a similar way on $\Omega^{\prime}$ and $\Omega^{\prime \prime}$. Using Fubini's theorem as in $\left[1\right.$, p. 313] and the fact that for each $\omega^{\prime} \in \Omega^{\prime}$ and each $\omega^{\prime \prime} \in \Omega^{\prime \prime}$, $\xi_{k}^{\prime \prime}\left(\omega^{\prime \prime}\right) \xi_{n}(\omega)$ and $\epsilon_{k}^{\prime}\left(\omega^{\prime}\right) \epsilon_{n}(\omega)$ are similar, respectively, to $\xi_{n}(\omega)$ and $\epsilon_{n}(\omega)$, we obtain that, almost surely with respect to $\Omega \times \Omega^{\prime} \times \Omega^{\prime \prime}$

$$
\sum_{k=1}^{\infty} \epsilon_{k}^{\prime} U_{k}\left(\xi_{k}^{\prime \prime} x\right) \in L^{\infty}
$$

and

$$
\underset{p \rightarrow \infty}{\limsup }\left\|\sum_{k=1}^{p} \epsilon_{k}^{\prime} U_{k}\left(\xi_{k}^{\prime \prime} x\right)\right\|_{\infty}<\infty .
$$

The events $\left\|U_{k}(x)\right\|_{\infty} \geqq \epsilon$ are independent, therefore almost surely infinitely many of them are simultaneously verified. This implies that for almost every $\omega \in \Omega$ we can find a sequence $k_{j}$ such that $\left\|U_{k_{j}}\right\|_{\infty} \geqq \epsilon$. Also for almost every $\omega \in \Omega$ (8) and (9) hold with probability one with respect to $\Omega^{\prime} \times \Omega^{\prime \prime}$. We fix now $\omega \in \Omega$ so that (8) and (9) hold almost surely with respect to $\Omega^{\prime} \times \Omega^{\prime \prime}$ and infinitely many open sets $I_{j}=\left\{x \in G:\left|U_{k_{j}}(x)\right|>\epsilon / 2\right\}$ are nonempty. This $\omega \in \Omega$ will remain fixed in what follows. Let $J_{j}=\left(\xi_{j}^{\prime \prime}\right)^{-1} I_{j}$. The $\Omega^{\prime \prime}$-event $J_{j} \neq \varnothing$ has probability one and because of the independence of $\xi_{k j}^{\prime \prime}$, almost surely we can find a point of $G$ which belongs to infinitely many $J_{j}$. We choose then $\omega^{\prime \prime}$ so that (9) holds almost surely with respect to $\Omega^{\prime}$ and a point $x_{0}$ belongs to infinitely many $J_{j}$. But if (9) holds almost surely, in particular,

$$
\underset{p \rightarrow \infty}{\limsup }\left|\sum_{k=1}^{p} \epsilon_{k}^{\prime} U_{k}\left(\xi_{k}^{\prime \prime} x_{0}\right)\right|<\infty
$$

almost surely (with respect to $\Omega^{\prime} ; U_{k}\left(\xi_{k}^{\prime \prime} x_{0}\right)$ are now constants since $\omega$ and $\omega^{\prime \prime}$ have been fixed). This implies by [6, (8.3), p. 213 and Remark (d), p. 205] that $\sum\left|U_{k}\left(\xi_{k}^{\prime \prime} x_{0}\right)\right|^{2}<\infty$ which contradicts the fact that for infinitely many $k,\left|U_{k}\left(\xi_{k} x_{0}\right)\right|>\epsilon / 2$.

REMARK 1. The conclusion of the theorem also holds for random Fourier series of the type $\sum d_{i} \operatorname{tr}\left(X_{i} D_{i}(x)\right)$ where $X_{i}$ are independent random variables such that $X_{i}$ is similar to $-X_{i}$ and to $X_{i} D_{i}(x)$ for each $x \in G$. A proof can be obtained using Fubini's theorem as in [2, Théorème 6].

REMARK 2. In the commutative case the hypothesis that $\sum \epsilon_{i} d_{i} \operatorname{tr}\left(A_{i} D_{i}\left(\xi_{i} x\right)\right)$ represents a bounded function for every choice of $\xi_{i} \in G$ and $\epsilon_{i}= \pm 1$ is sufficient to imply that the series represents a 
function belonging to $L^{2}(G) * L^{2}(G)$. The example given in [3] shows that this may be false in the noncommutative case.

3. A counterexample. As remarked in the Introduction, the conclusion of the theorem holds for random Fourier series, defined on a commutative compact group $G$, of the type

$$
\sum_{n=1}^{\infty} \epsilon_{n} a_{n} \gamma_{n}(x)
$$

where $\gamma_{n}$ are characters of $G$ and $a_{n}$ fixed complex numbers. This fact is an immediate consequence of Theorem 6 of [5, Chapter II] or of the Corollary to the Theorem 3 of [4].

For noncommutative groups the situation is entirely different: in this section we shall construct an example of a compact group $G$ and a series

$$
\sum_{i=1}^{\infty} \epsilon_{i} \operatorname{tr}\left(A_{i} D_{i}(x)\right)
$$

which represents for every value of $\epsilon_{i}$ a bounded function and yet for no such value it represents a continuous function. We let $H$ be the dihedral group which we represent faithfully as the group of two by two matrices of the type

$$
\left(\begin{array}{ll}
\epsilon_{1} & 0 \\
0 & \epsilon_{2}
\end{array}\right) \text { or }\left(\begin{array}{ll}
0 & \epsilon_{1} \\
\epsilon_{2} & 0
\end{array}\right) \text { with } \epsilon_{1}, \epsilon_{2}= \pm 1 .
$$

We let $H_{n}, n=1,2, \cdots$, be copies of $H$ and $G=\prod_{n=1}^{\infty} H_{n}$. Let $D_{n}: G \rightarrow H_{n}$ be the irreducible representation of degree two obtained by projecting $G$ onto $H_{n}$. Thus if $x \in G, x=\left\{x_{n}\right\}, x_{n} \in H_{n}$, then $D_{n}(x)=x_{n}$. Let $v_{1}^{(n)}(x)$ be the entry in the upper left corner of the matrix $x_{n}$ and $v_{2}^{(n)}(x)$ be the entry in the upper right corner. Define

$$
\begin{aligned}
& f_{1}(x)=v_{1}^{(1)}(x), \\
& f_{2}(x)=v_{2}^{(1)}(x) v_{1}^{(2)}(x), \\
& \cdots, \\
& f_{n}(x)=v_{2}^{(1)}(x) v_{2}^{(2)}(x) \cdots v_{1}^{(n)}(x) .
\end{aligned}
$$

Then since for each $k, v_{1}^{(k)}(x) v_{2}^{(k)}(x) \equiv 0, f_{n}(x) f_{m}(x) \equiv 0$ for $n \neq m$. Moreover each $f_{n}$ belongs to the translation invariant subspace of $L^{2}$ generated by the character of the representation $\widetilde{D}_{n}=D_{1} \otimes D_{2} \otimes$ $\cdots \otimes D_{n}$. Now the representations $\tilde{D}_{n}$ are all irreducible, therefore 
the series

$$
\sum_{n=1}^{\infty} \epsilon_{n} f_{n}
$$

is a Fourier series of the type of (10). We also have that

$$
\sup _{p}\left\|\sum_{n=1}^{p} \epsilon_{n} f_{n}\right\|_{\infty} \leqq 1,
$$

because for each $x$ only one of the $f_{n}$ will be such that $f_{n}(x) \neq 0$. Therefore (11) represents a bounded measurable function for every choice of the $\epsilon_{n}$. On the other hand it is easy to see that (11) cannot represent a continuous function $f$ for any value of $\epsilon_{n}$. Indeed if $\epsilon_{n}$ are fixed we define sequences $x_{N}$ and $y_{N}$ of element of $G$ by letting

$$
\begin{gathered}
D_{n}\left(x_{N}\right)=D_{n}\left(y_{N}\right)=\left(\begin{array}{ll}
0 & 1 \\
1 & 0
\end{array}\right) \text { if } n<N, \\
D_{n}\left(x_{N}\right)=\left(\begin{array}{ll}
1 & 0 \\
0 & 1
\end{array}\right) \text { and } D_{n}\left(y_{N}\right)=\left(\begin{array}{rr}
-1 & 0 \\
0 & -1
\end{array}\right) \text { if } n \geqq N ;
\end{gathered}
$$

we have that $\lim _{N} y_{N}=\lim _{N} x_{N}$, but $f\left(x_{N}\right)=f_{N}\left(x_{N}\right)=\epsilon_{N}$ and $f\left(y_{N}\right)$ $=f_{N}\left(y_{N}\right)=-\epsilon_{N}$. Therefore $f$ cannot be continuous.

\section{REFERENCES}

1. P. Billard, Séries de Fourier aléatoirement bornées, continues, uniformément convergentes, Studia Math. 22 (1963), 309-330.

2. - Séries de Fourier aléatoirement bornées, continues, uniformément convergentes, Ann. Ecole Norm. Sup. (3) 82 (1965), 131-179.

3. A. Figà-Talamanca, Appartenenza a $L^{p}$ delle series di Fourier aleatorie su gruppi non commutativi, Rend. Sem. Mat. Univ. Padova 39 (1967), 330-348.

4. J.-P. Kahane, Sur les sommes vectorielles $\sum \pm u_{n}$ C. R. Acad. Sci. Paris 259 (1964), 2577-2580.

5. - Some random series of functions, Heath, Boston, Mass., 1968.

6. A. Zygmund, Trigonometric series, Vol. I, 2nd ed., Cambridge Univ. Press, New York, 1959.

University of California, Berkeley 